\title{
Prior cardiovascular disease increases long-term mortality in COPD patients with pneumonia
}

\author{
Oriol Sibila ${ }^{1,2}$, Eric M. Mortensen ${ }^{3,4}$, Antonio Anzueto ${ }^{1,5}$, Elena Laserna ${ }^{1,6}$ and \\ Marcos I. Restrepo ${ }^{1,5,7}$
}

\author{
Affiliations: \\ 'University of Texas Health Science Center at San Antonio, San Antonio, TX, \\ ${ }^{3}$ Veterans Affairs North Texas Health Care System, San Antonio, TX, \\ ${ }^{4}$ University of Texas Southwestern Medical Center, Dallas, TX, \\ ${ }^{5}$ South Texas Veterans Health Care System, San Antonio, TX, and \\ ${ }^{7}$ Veterans Evidence Based Research Dissemination and Implementation Center (VERDICT), San Antonio, \\ TX, USA. \\ ${ }^{2}$ Servei de Pneumologia, Hospital de la Santa Creu i Sant Pau, Barcelona, and \\ ${ }^{6}$ Hospital Comarcal de Mollet, Mollet del Valles, Spain.
}

Correspondence: M.I. Restrepo, VERDICT (11C6), South Texas Veterans Health Care System ALMD, 7400 Merton Minter Boulevard, San Antonio, TX, 78229, USA. E-mail: restrepomduthscsa.edu

ABSTRACT There is controversy regarding the impact of chronic obstructive pulmonary disease (COPD) in clinical outcomes in elderly patients with pneumonia. Comorbidities such as cardiovascular disease have been reported to play an important role in patients with acute exacerbations of COPD. However, limited data are available regarding the impact of cardiovascular disease in elderly COPD patients who require hospitalisation for pneumonia.

We examined a cohort of subjects with pneumonia and pre-existing COPD. Prior cardiovascular disease was defined as history of myocardial infarction, congestive heart failure, cardiac arrhythmia, unstable angina or stroke. Outcomes examined included 30-day, 90-day, 6-month and 1-year mortality.

We included 17140 elderly COPD patients who were hospitalised for pneumonia. Prior cardiovascular disease was present in 10240 (59.7\%) patients. Prior cardiovascular disease was independently associated with 90 -day mortality ( $21.3 \%$ versus $19.4 \%$; hazard ratio (HR) $1.29,95 \%$ CI $1.02-1.17$ ), 6 -month mortality (29.0\% versus $26.1 \%$; HR 1.28 , 95\% CI $1.07-1.50)$ and 12 -month mortality (39.2\% versus $34.5 \%$; HR 1.33 , 95\% CI 1.15-1.54) when compared to no prior cardiovascular disease. The temporal differential effect between groups increases from $1.0 \%$ at 30 days to $4.7 \%$ at 1 year.

Prior cardiovascular disease is associated with increased long-term mortality in elderly COPD patients with pneumonia. Differences in mortality rates increased over time.

@ERSpublications

Prior cardiovascular disease is associated with increased long-term mortality in elderly COPD patients with pneumonia http://ow.ly/qaAJY

Received: July 262012 | Accepted after revision: March 222013 | First published online: April 182013

Support statement: This research was supported by Howard Hughes Medical Institute faculty-start up grant 00378-001 and a Dept of Veteran Affairs Veterans Integrated Service Network 17 new faculty grant. O. Sibila is supported by Instituto de Salud Carlos III (BAE11/00102). O. Sibila and E. Laserna are supported by Sociedad Espanola de Neumologia y Cirugia Toracica (SEPAR), Societat Catalana de Pneumologia (SOCAP) and Fundacio Catalana de Pneumologia (FUCAP). M.I. Restrepo's time is partially protected by Award Number K23HL096054 from the National Heart, Lung, and Blood Institute. The funding agencies had no role in the preparation, review, or approval of the manuscript. The views expressed in this article are those of the author and do not necessarily represent the views of the Dept of Veterans Affairs.

Conflict of interest: Disclosures can be found alongside the online version of this article at www.erj.ersjournals.com 


\section{Introduction}

Chronic obstructive pulmonary disease (COPD) is the third leading cause of death in the USA, affecting $>12$ million people [1]. COPD is characterised by progressive airflow limitation and intermittent episodes of exacerbations that are mainly caused by viral or bacterial infections [2]. Although COPD is a lung disease, comorbid conditions play an important role in the natural history of the disease [3]. The most common comorbid condition in COPD patients is cardiovascular disease $[4,5]$. Furthermore, COPD patients with cardiovascular disease who develop an acute exacerbation have higher mortality $[6,7]$.

It is not clear whether COPD patients who develop pneumonia have worse outcomes than those without COPD. Studies have shown that COPD patients who develop pneumonia have higher mortality [8-10], a protective effect $[11]$ or no effect $[12,13]$. These differences in mortality may be associated with the comorbid conditions rather than the pneumonia itself [14]. However, limited data are available regarding the impact of both pre-existing cardiovascular disease and COPD in elderly patients hospitalised with pneumonia.

Therefore, the aim of the present study was to examine the impact of prior cardiovascular disease in shortand long-term mortality in COPD patients hospitalised with pneumonia. Our hypothesis was that patients with COPD with an acute infectious process such as pneumonia will have significantly higher mortality if prior cardiovascular disease is present.

\section{Methods}

We conducted a retrospective cohort study using the national administrative database of the Dept of Veterans Affairs (VA) Health Care System to examine outcomes for elderly COPD patients hospitalised with pneumonia. The VA databases are the repositories of clinical and administrative data from the $>150$ VA hospitals and 850 clinics. The institutional review board of the University of Health Science Center at San Antonio (San Antonio, TX, USA) approved this study.

\section{Inclusion and exclusion criteria}

We identified all patients who had a VA hospital stay during fiscal years 2002-2007 (October 2001 to September 2007) with a prior diagnosis of COPD (International Classification of Diseases (ICD), ninth revision codes 490-492 or 496) and a primary discharge diagnosis of pneumonia (ICD-9 codes 480.0-483.99 or 485-487.0) or a secondary discharge diagnosis of pneumonia with a primary diagnosis of respiratory failure (ICD-9 code 518.81) or sepsis (ICD-9 code 038.xx).

We included elderly patients in this study if they 1) were aged $\geqslant 65$ years on the date of hospital presentation; 2) had $\geqslant 1$ year of VA outpatient care before admission; 3 ) had received at least one of the following respiratory medications during the 30 -day period before hospitalisation: any form of $\beta$-agonist, theophylline, tiotropium or ipratropium bromide; and 4) had received at least one dose of antibiotics within $48 \mathrm{~h}$ of admission. For patients with multiple hospitalisations, we only assessed the first admission during the study period.

We excluded patients with history of asthma (ICD-9 codes 493.xx).

Data

This study used demographic, utilisation and clinical data from the National Patient Care Database, pharmacy data from the VA Decision Support System and Pharmacy Benefits Management group, and mortality data from the VA vital status file. Encrypted patient identifiers were used to link the information from each database.

We obtained demographic information (age, sex, race and marital status) from in-patient and outpatient data. We categorised race as white, black, other or missing. We used ICD-9 codes for tobacco use (305.1 and V15.82), attendance at a smoking cessation clinic or use of medications for the treatment of nicotine dependence (Zyban (GlaxoSmithKline, Brentford, UK), nicotine replacement or varenicline) to identify recent tobacco use.

We assessed the presence of comorbid conditions using the Charlson-Deyo system to assign comorbidity scores for pre-existing conditions [15]. The Charlson-Deyo comorbidity score is based on 19 comorbid conditions, and we included the individual conditions in the survival models.

The primary independent variable of interest was prior cardiovascular disease. We identified cardiovascular events in the cohort using ICD-9 codes indicating diagnosis of myocardial infarction, congestive heart failure, cardiac arrhythmia, unstable angina and stroke as previously described [16]. We defined prior cardiovascular events as those that occurred $\geqslant 1$ day before the day of admission for the pneumonia hospitalisation and examined records dating back to $\geqslant 1$ year before that hospitalisation. 


\section{Outcomes}

The primary outcomes for this study were 30-day, 90-day, 6-month and 12-month mortality. We chose these periods for follow-up because previous research demonstrated that mortality within 30 days is primarily pneumonia-related, whereas mortality after 60 days is more frequently caused by comorbid conditions [14]. Mortality was assessed to January 1, 2008, using the VA vital status file. Previous studies have demonstrated that this data source has a sensitivity of $\sim 98 \%$ for veterans' deaths [17].

\section{Statistical analysis}

We used bivariate statistics to test the associations of demographics and clinical characteristics and mortality. Categorical variables were analysed using t-tests. We defined statistical significance using a twotailed p-value $<0.05$. We performed a multivariable analysis using a Cox proportional survival model examining mortality over the year after admission. We also present a Kaplan-Meier curve representing the survival data. Potential confounders (with p-values $<0.1$ in the univariate analyses) included in the regression models of mortality were age, race, marital status, smoker, alcohol abuse, peripheral vascular disorder, hemiplegia, diabetes (complicated and uncomplicated), malignancy, metastatic cancer, cerebrovascular disease and cardiovascular or diabetes medications.

All analyses were performed using SPSS version 19.0 for Windows (SPSS Inc; Chicago, IL, USA).

\section{Results}

A total of 17140 elderly patients meet all study eligibility criteria. Of these, 10240 (59.7\%) had prior cardiovascular disease and $6900(40.3 \%)$ had no prior cardiovascular disease. The more frequent cardiovascular conditions were congestive heart failure $(n=6673,65.1 \%)$, cardiac arrhythmia $(n=6244,60.9 \%)$, myocardial infarction $(n=3020,29.5 \%)$ and unstable angina $(n=2994,29.2 \%)$. Our cohort of pneumonia patients with COPD were mainly male (98.5\%), elderly (mean \pm SD age $76.4 \pm 6.3$ years) and married (54.4\%). Comparisons of patient characteristics stratified by the presence or absence of prior cardiovascular disease showed that those with prior cardiovascular disease were less likely to have cancer, current tobacco use or alcohol abuse, and more likely to be married, have diabetes or renal disease, or to be receiving cardiovascular or diabetes medications. There were no statistically significant differences regarding pulmonary medications, including inhaled corticosteroids among cardiovascular risk groups (table 1).

Overall, of the COPD patients hospitalised with pneumonia, 2045 (11.9\%) died within 30 days, 3506 (20.5\%) within 90 days, $4769(27.8 \%)$ within 6 months and 6392 (37.3\%) within 12 months. In the unadjusted model, COPD patients with prior cardiovascular disease hospitalised with pneumonia had significantly higher 30 -day mortality ( $12.3 \%$ versus $11.3 \%)$, 90 -day mortality $(21.1 \%$ versus $19.4 \%)$, 6-month mortality $(29.0 \%$ versus $26.1 \%$ ) and 12 -month mortality ( $39.2 \%$ versus $34.5 \%$ ), when compared to patients without prior cardiovascular disease (table 2). Furthermore, in the Cox proportional survival multivariable analyses, after adjusting for potential confounders, prior cardiovascular disease remains independently associated with mortality at 90 days (hazard ratio (HR) 1.29, 95\% CI 1.02-1.17), 6 months (HR 1.28, 95\% CI 1.07-1.50) and 12 months (HR 1.33, 95\% CI 1.15-1.54) (table 2). Figure 1 shows a Kaplan-Meier curve representing the survival data. Temporal differential mortality effect between groups increases from $1.0 \%$ at 30 days to $4.7 \%$ at 1 year among COPD patients hospitalised with communityacquired pneumonia (CAP) (fig. 2).

\section{Discussion}

Our study suggests that prior cardiovascular disease in elderly COPD patients hospitalised with pneumonia is associated with higher long-term mortality as compared to those without prior cardiovascular disease. In addition, the temporal differential mortality effect among groups increases over time. To our knowledge, no other study has evaluated the impact of prior cardiovascular disease in short- and long-term mortality among COPD patients who are hospitalised with pneumonia.

Comorbidities are recognised to have an important impact on the management and prognosis of COPD [3]. In addition to smoking, elderly patients with COPD have other risk factors for cardiovascular disease, due to their advanced age and reduced levels of physical activity. Several studies have recognised cardiovascular disease as the most common comorbid condition in COPD patients [4, 5]. In our cohort, nearly $60 \%$ of the COPD patients had a concomitant comorbid diagnosis of prior cardiovascular disease at the time of pneumonia presentation. Furthermore, previous studies also detected cardiovascular disease as the most common condition in pneumonia $[18,19]$. In addition, different studies $[20,21]$ have showed an increased risk of mortality among COPD patients with cardiovascular disease. Similar results have been observed among patients with pneumonia and cardiovascular disease [22, 23]. Therefore, a better understanding of the 
TABLE 1 Patient demographic and clinical characteristics by the presence of prior cardiovascular (CV) disease

No prior CV disease

$\begin{array}{lc}\text { Subjects } & 6900(40.3) \\ \text { Age years } & 75.3 \pm 6.3 \\ \text { Male } & 6785(98.3) \\ \text { Race/ethnicity } & \\ \text { White } & 5967(86.5) \\ \text { Black } & 587(8.5) \\ \text { Other } & 345(5.0) \\ \text { Missing } & 279(4.0) \\ \text { Married } & 3680(53.3) \\ \text { Smoker } & 3548(51.4) \\ \text { Alcohol abuse } & 369(5.3) \\ \text { Drug abuse } & 85(1.2) \\ \text { Pre-existing comorbid conditions } & \\ \text { Peripheral vascular disorders } & 853(12.4) \\ \text { Hemiplegia or paraplegia } & 77(1.1) \\ \text { Diabetes } & \\ \quad \text { Uncomplicated } & 1382(20.0) \\ \quad \text { Complicated } & 300(4.3) \\ \text { Renal disease } & 405(5.9) \\ \text { Moderate or severe liver disease } & 49(0.7) \\ \text { Mild liver disease } & 18(0.3) \\ \text { Peptic ulcer disease } & 237(3.4) \\ \text { AlDS } & 14(0.2) \\ \text { Metastatic cancer } & 288(4.2) \\ \text { Malignancy } & 1919(27.8) \\ \text { Leukaemia } & 136(2.0) \\ \text { Rheumatoid arthritis and collagen vascular diseases } & 188(2.7) \\ \text { Cerebrovascular disease } & 850(12.3) \\ \text { Dementia } & 160(2.3) \\ \text { Medications } & \\ \text { Pulmonary medications } & 3.5 \pm 1.7 \\ \text { Cardiovascular medications } & 1.3 \pm 1.3 \\ \text { Diabetes medications } & 0.2 \pm 0.5\end{array}$

Prior CV disease

p-value

$10240(59.7)$

$76.8 \pm 6.4$

$10092(98.6)$

$<0.001$

0.25

8957 (87.5)

891 (8.7)

$392(3.8)$

$298(2.9)$

5649 (55.2)

4946 (48.3)

0.018

$426(4.2)$

$138(1.3)$

$<0.001$

0.536

1910 (18.7)

114 (1.1)

$<0.001$

1.0

$3462(33.8)$

1018 (9.9)

$1440(14.1)$

$72(0.7)$

$28(0.3)$

380 (3.7)

$13(0.1)$

339 (3.3)

$2502(24.4)$

175 (1.7)

302 (2.9)

1788 (17.5)

275 (2.7)

$<0.001$

$<0.001$

$<0.001$

1.0

0.996

0.358

0.241

0.004

$<0.001$

0.220

0.400

$<0.001$

0.138

$3.5 \pm 1.7$

$2.5 \pm 1.6$

$0.3 \pm 0.6$
0.467

$<0.001$

$<0.001$

Data are presented as $\mathrm{n}(\%)$ or mean $\pm \mathrm{SD}$, unless otherwise stated.

complexity of these interactions among chronic diseases (e.g. COPD and cardiovascular disease) and acute conditions (e.g pneumonia) may help clinicians to understand how to improve clinical outcomes for patients.

Several studies have shown that hospitalised pneumonia patients are associated with increased long-term mortality, but the potential reasons for these observations are unclear [22, 24]. MORTENSEN et al. [14] demonstrated that mortality within 30 days was associated with pneumonia and that within 30 and 90 days was not associated with pneumonia, but mainly with comorbid conditions. In addition, some authors have shown that an increasing number of comorbid conditions in pneumonia patients are associated with this

TABLE 2 Mortality assessment of chronic obstructive pulmonary disease patients hospitalised with community-acquired pneumonia according to prior cardiovascular disease status

\begin{tabular}{|c|c|c|c|c|c|c|}
\hline Mortality & \multicolumn{2}{|c|}{ Prior cardiovascular disease } & \multicolumn{2}{|c|}{ Unadjusted } & \multicolumn{2}{|c|}{ Adjusted $^{\#}$} \\
\hline 30-day & $782(11.3)$ & $1263(12.3)$ & $1.10(1.01-1.20)$ & 0.042 & $1.18(0.73-1.10)$ & 0.3 \\
\hline 6-month & $1800(26.1)$ & $2969(29.0)$ & $1.13(1.06-1.20)$ & $<0.001$ & $1.27(1.07-1.50)$ & 0.006 \\
\hline 12-month & 2380 (34.5) & 4012 (39.2) & $1.17(1.11-1.23)$ & $<0.001$ & $1.34(1.16-1.54)$ & $<0.001$ \\
\hline
\end{tabular}

Data are presented as $\mathrm{n}(\%)$, unless otherwise stated. HR: hazard ratio. ${ }^{\#}$ : potential confounders, with $\mathrm{p}$-values $<0.1$ in the univariate analyses, were age, race, marital status, smoker, alcohol abuse, peripheral vascular disorder, hemiplegia, diabetes (complicated and uncomplicated), malignancy, metastatic cancer, cerebrovascular disease and cardiovascular or diabetes medications. 


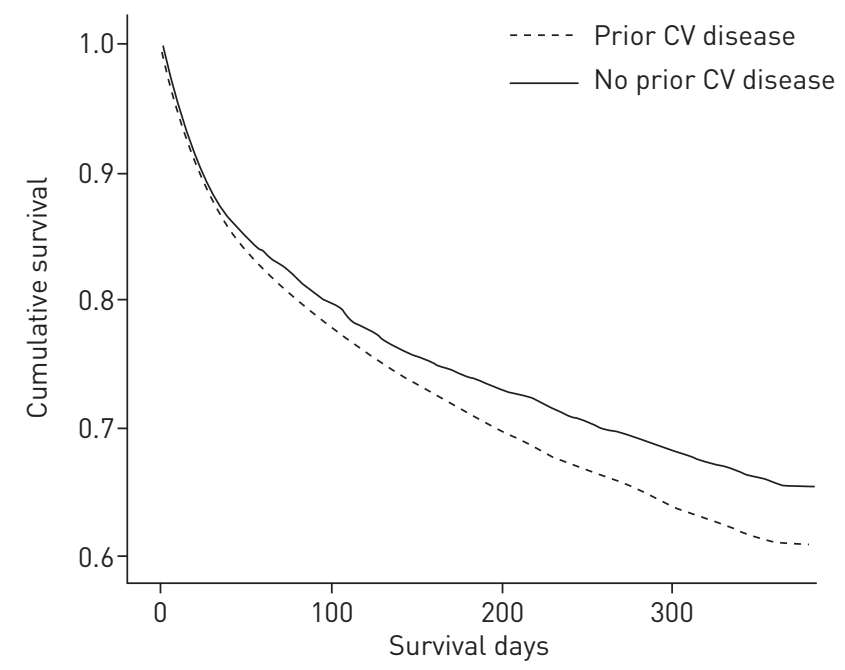

FIGURE 1 Kaplan-Meier curve among elderly chronic obstructive pulmonary disease patients hospitalised with communityacquired pneumonia according the presence of prior cardiovascular $(\mathrm{CV})$ disease.

increased long-term mortality [19, 22]. However, other studies suggest that the higher long-term mortality of pneumonia cannot be solely explained by higher burden of chronic comorbid conditions predating the occurrence of pneumonia [18]. Pneumonia may accelerate progression of pre-existing chronic inflammatory diseases. Recent studies have shown that acute infections such as pneumonia may trigger further inflammatory deleterious effects [25], which may be associated with poor clinical outcomes. Higher concentrations of inflammatory markers in blood, such as C-reactive protein, procalcitonin and white blood cells, have been shown to precede the development of acute coronary syndromes [26, 27]. Acute infections, in addition to eliciting systemic inflammatory responses, can also have direct effects on atherosclerotic plaques and coronary arteries [28, 29] and may worsen the long-term prognosis of cardiovascular diseases. Our results suggest that worsening of prior cardiovascular disease may be the factor associated with higher mortality. Our data showed that having prior cardiovascular disease is independently associated with increased long-term mortality among elderly COPD patients hospitalised with pneumonia. In addition, there is a temporal differential incremental mortality effect.

Although our study was a large database analysis and subject to the recognised limitations of such studies, we carefully assembled our cohort from complete patient discharge data to avoid ascertainment bias. Our sample was predominantly elderly and male because of our use of VA administrative data, and it is possible that females with COPD and younger groups may have a different natural history. Several variables were not available in this secondary data analysis, including pulmonary function tests to assess the severity of the disease, the severity and number of cardiovascular events per year of disease and the use of systemic corticosteroid treatment during hospitalisation. In addition, we could not differentiate between CAP and healthcare-associated pneumonia among patients admitted with pneumonia. Another important limitation was reliance on ICD-9 codes for the diagnosis of COPD and cardiovascular disease However, in order to adjust for the appropriate ICD-9 diagnosis, we relied on validated tools by PERRY et al. [16], JOo et al. [30] and COOKE et al. [31], which use specific pulmonary medications recommended for COPD patients. In addition, we used the number of diabetes and cardiovascular medications in the multivariate disease-state

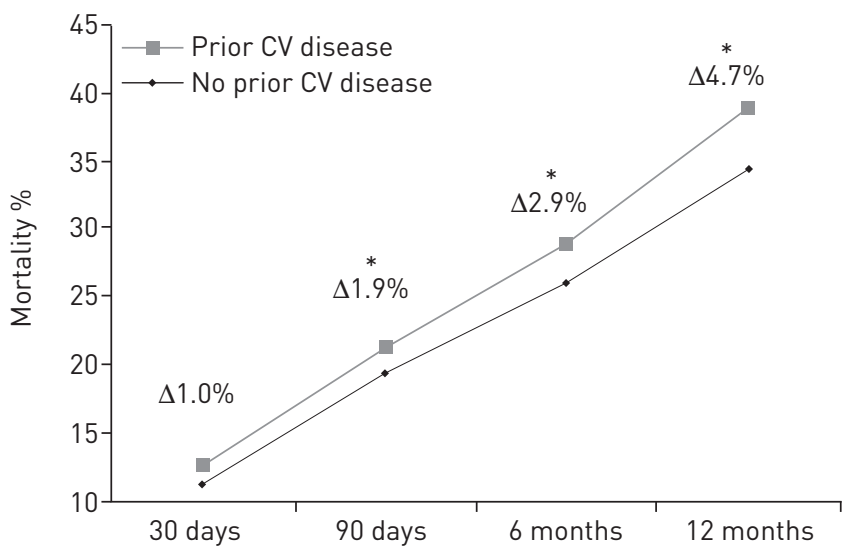

FIGURE 2 Temporal differential mortality effect $(\Delta)$ among elderly chronic obstructive pulmonary disease patients hospitalised with community-acquired pneumonia according to prior cardiovascular (CV) disease status. ${ }^{\star}: \mathrm{p}<0.05$. 
analysis to control for possible confounders that may affect clinical outcomes. Our study is limited in its ability to examine the causes of death and the mechanisms by which acute inflammation in patients with pneumonia may increase mortality in elderly patients with prior COPD and cardiovascular disease. Further studies should use a different design in order to conclusively demonstrate that this increase in mortality is due to cardiac events.

In conclusion, we suggest that prior cardiovascular disease was associated with higher 90-day, 6-month and 12-month mortality in elderly patients with COPD who were hospitalised with pneumonia, after adjusting for potential confounders. Despite the intrinsic limitations of observational studies using ICD-9 codes, these findings should serve as hypothesis-generating data and contribute to the solution of ongoing controversies. Clinicians should consider intensive treatment and control of cardiovascular disease in elderly COPD patients after an acute episode of pneumonia in order to improve their long-term survival. Additional studies are needed to understand the underlying mechanisms that are responsible for these findings.

\section{References}

1 Miniño AM, Murphy SL, Xu J, et al. Deaths: final data for 2008. Natl Vital Stat Rep 2011; 59: 1-126.

2 Anzueto A, Sethi S, Martinez FJ. Exacerbations of chronic obstructive pulmonary disease. Proc Am Thorac Soc 2007; 4: $554-564$.

3 Agustí A, Barnes PJ. Update in chronic obstructive pulmonary disease 2011. Am J Respir Crit Care Med 2012; 185: 1171-1176.

4 Holguin F, Folch E, Redd SC, et al. Comorbidity and mortality in COPD-related hospitalizations in the United States, 1979 to 2001. Chest 2005; 128: 2005-2011.

5 Curkendall SM, DeLuise C, Jones JK, et al. Cardiovascular disease in patients with chronic obstructive pulmonary disease, Saskatchewan Canada cardiovascular disease in COPD patients. Ann Epidemiol 2006; 16: 63-70.

6 Dewan NA, Rafique S, Kanwar B, et al. Acute exacerbation of COPD: factors associated with poor treatment outcome. Chest 2000; 117: 662-671.

$7 \quad$ Agabiti N, Belleudi V, Davoli M, et al. Profiling hospital performance to monitor the quality of care: the case of COPD. Eur Respir J 2010; 35: 1031-1038.

8 Restrepo MI, Mortensen EM, Pugh JA, et al. COPD is associated with increased mortality in patients with community-acquired pneumonia. Eur Respir J 2006; 28: 346-351.

9 Rello J, Rodriguez A, Torres A, et al. Implications of COPD in patients admitted to the intensive care unit by community-acquired pneumonia. Eur Respir J 2006; 27: 1210-1216.

10 Molinos L, Clemente MG, Miranda B, et al. Community-acquired pneumonia in patients with and without chronic obstructive pulmonary disease. J Infect 2009; 58: 417-424.

11 Menéndez R, Torres A, Zalacaín R, et al. Risk factors of treatment failure in community acquired pneumonia: implications for disease outcome. Thorax 2004; 59: 960-965.

12 Torres A, Dorca J, Zalacaín R, et al. Community-acquired pneumonia in chronic obstructive pulmonary disease: a Spanish multicenter study. Am J Respir Crit Care Med 1996; 154: 1456-1461.

13 Liapikou A, Polverino E, Ewig S, et al. Severity and outcomes of hospitalised community-acquired pneumonia in COPD patients. Eur Respir J 2012; 39: 855-861.

14 Mortensen EM, Coley CM, Singer DE, et al. Causes of death for patients with community-acquired pneumonia: results from the Pneumonia Patient Outcomes Research Team cohort study. Arch Intern Med 2002; 162: 1059-1064.

15 Charlson M, Szatrowski TP, Peterson J, et al. Validation of a combined comorbidity index. J Clin Epidemiol 1994; 47: 1245-1251.

16 Perry TW, Pugh MJ, Waterer GW, et al. Incidence of cardiovascular events after hospital admission for pneumonia. Am Med J 2011; 124: 244-251.

17 Sohn MW, Arnold N, Maynard C, et al. Accuracy and completeness of mortality data in the Department of Veterans Affairs. Popul Health Metr 2006; 4: 2.

18 Yende S, Angus DC, Ali IS, et al. Influence of comorbid conditions on long-term mortality after pneumonia in older people. J Am Geriatr Soc 2007; 55: 518-525.

19 Waterer GW, Lessler LA, Wunderink RG. Medium-term survival after hospitalization with community-acquired pneumonia. Am J Respir Crit Care Med 2004; 169: 910-914.

20 Macchia A, Monte S, Romero M, et al. The prognostic influence of chronic obstructive pulmonary disease in patients hospitalised for chronic heart failure. Eur J Heart Fail 2007; 9: 942-948.

21 Sidney S, Sorel M, Quesenberry CP Jr, et al. COPD and incident cardiovascular disease hospitalizations and mortality: Kaiser Permanente Medical Care Program. Chest 2005; 128: 2068-2075.

22 Mortensen EM, Kapoor WN, Chang CC, et al. Assessment of mortality after long-term follow-up of patients with community-acquired pneumonia. Clin Infect Dis 2003; 37: 1617-1624.

23 Kaplan V, Angus DC, Griffin MF, et al. Hospitalized community-acquired pneumonia in the elderly: age- and sexrelated patterns of care and outcomes in the United States. Am J Respir Crit Care Med 2002; 165: 766-772.

24 Mortensen EM. Potential causes of increased long-term mortality after pneumonia. Eur Respir J 2011; 37: 1306-1307.

25 Corrales-Medina VF, Madjid M, Musher DM. Role of acute infection in triggering acute coronary syndromes. Lancet Infect Dis 2010; 10: 83-92.

26 Liuzzo G, Biasucci LM, Gallimore JR, et al. The prognostic value of C-reactive protein and serum amyloid a protein in severe unstable angina. N Engl J Med 1994; 331: 417-424.

27 Kafkas N, Venetsanou K, Patsilinakos S, et al. Procalcitonin in acute myocardial infarction. Acute Card Care 2008; 10: $30-36$.

28 Naghavi M, Wyde P, Litovsky S, et al. Influenza infections exerts prominent and thrombotic effects on the atherosclerotic plaques of apolipoprotein E-deficient mice. Circulation 2003; 107: 762-768. 
29 Madjid M, Vela D, Khalili-Tabrizi H, et al. Systemic infections cause exaggerated inflammation in atherosclerotic coronary arteries: clues to the triggering effect of acute infections on acute coronary syndromes. Tex Heart Inst J 2007; 34: 11-18.

30 Joo MJ, Lee TA, Au DH, et al. Medication use patterns associated with spirometry in diagnosing COPD. COPD 2008; 5: 360-368

31 Cooke CR, Joo MJ, Anderson SM, et al. The validity of using ICD-9 codes and pharmacy records to identify patients with chronic obstructive pulmonary disease. BMC Health Serv Res 2011; 11: 37. 